\title{
Inhibition of nitric oxide synthesis during induced cholestasis ameliorates hepatocellular injury by facilitating S-nitrosothiol homeostasis
}

\author{
Laura M López-Sánchez ${ }^{1}$, Fernando J Corrales ${ }^{2}$, Montserrat Barcos ${ }^{3}$, Isabel Espejo ${ }^{3}$, Juan R Muñoz-Castañeda ${ }^{1}$ \\ and Antonio Rodríguez-Ariza ${ }^{1}$
}

Cholestatic liver injury following extra- or intrahepatic bile duct obstruction causes nonparenchymal cell proliferation and matrix deposition leading to end-stage liver disease and cirrhosis. In cholestatic conditions, nitric oxide (NO) is mainly produced by a hepatocyte-inducible NO synthase (iNOS) as a result of enhanced inflow of endotoxins to the liver and also by accumulation of bile salts in hepatocytes and subsequent hepatocellular injury. This study was aimed to investigate the role of NO and S-nitrosothiol (SNO) homeostasis in the development of hepatocellular injury during cholestasis induced by bile duct ligation (BDL) in rats. Male Wistar rats $(200-250 \mathrm{~g})$ were divided into four groups $(n=10$ each), including sham-operated (SO), bile duct-ligated (BDL), tauroursodeoxycholic acid (TUDCA, $50 \mathrm{mg} / \mathrm{kg}$ ) and S-methylisothiourea (SMT, $25 \mathrm{mg} / \mathrm{kg}$ ) treated. After 7 days, BDL rats showed elevated serum levels of $\gamma$-glutamiltranspeptidase, aspartate aminotransferase, alanine aminotransferase, $\mathrm{LDH}$, and bilirubin, bile duct proliferation and fibrosis, compared with the SO group. TUDCA treatment did not significantly alter these parameters, but the iNOS inhibitor SMT ameliorated hepatocellular injury, as shown by lower levels of circulating hepatic enzymes and bilirubin, and a decreased grade of bile duct proliferation and fibrosis. Both TUDCA and SMT treatments reversed Mrp2 canalicular pump expression to control levels. However, only SMT treatment significantly lowered the increased levels of plasma NO and S-nitrosation (S-nitrosylation) of liver proteins in $\mathrm{BDL}$ rats. Moreover, $\mathrm{BDL}$ resulted in a reduction of the S-nitrosoglutathione reductase (GSNOR/Adh5) enzymatic activity and a downregulation of the GSNOR/Adh5 mRNA expression that was reverted by SMT, but not TUDCA, treatment. A total of 25 liver proteins, including S-adenosyl methionine synthetase, betainehomocysteine S-methyltransferase, Hsp90 and protein disulfide isomerase, were found to be S-nitrosated in BDL rats. In conclusion, the inhibition of NO production during induced cholestasis ameliorates hepatocellular injury. This effect is in part mediated by the improvement of cell proficiency in maintaining SNO homeostasis.

Laboratory Investigation (2010) 90, 116-127; doi:10.1038/labinvest.2009.104; published online 5 October 2009

KEYWORDS: Adh5; cholestasis; GSNOR; NO; S-nitrosoglutahione; S-nitrosylation

Cholestasis, an impairment or cessation in the flow of bile, occurs in many human diseases including sepsis, drug toxicity, viral hepatitis and cholangiopathies, although treatment options are limited. Hepatocellular injury due to the buildup of bile acids and other toxins in the liver is an invariant feature of cholestasis, promoting further hepatic dysfunction culminating in liver fibrosis, cirrhosis, portal hypertension and chronic liver failure. Optimal therapy would address the mechanisms causing cholestasis and reverse the process, but unfortunately such therapies do not currently exist.
Therefore, better understanding of the mechanisms of cholestatic hepatocellular injury could help to provide rational alternative therapeutic strategies to reduce hepatocellular damage. ${ }^{1}$ Several biochemical alterations, including toxic bile salts accumulation and oxidative alterations in the mitochondria, participate in the generation and development of cholestatic liver injury. ${ }^{2}$ Oxidative stress has an important role in hepatocyte cell death and hepatocellular injury during experimental induced cholestasis. ${ }^{3}$ Actually, bile acids have been reported to stimulate the generation of reactive oxygen

\footnotetext{
${ }^{1}$ Unidad de Investigación del Hospital Universitario Reina Sofía, Instituto Maimónides de Investigación Biomédica, Córdoba, Spain; ${ }^{2}$ Hepatology and Gene Therapy Unit, Universidad de Navarra, Pamplona, Spain and ${ }^{3}$ Servicio de Análisis Clínicos del Hospital Reina Sofía, Córdoba, Spain Correspondence: Dr A Rodríguez-Ariza, PhD, Unidad de Investigación, Hospital Universitario Reina Sofía, Avda Menéndez Pidal s/n, Córdoba 14004, Spain. E-mail: antonio.rodriguez.exts@juntadeandalucia.es

Received 22 June 2009; revised 14 August 2009; accepted 1 September 2009
} 
species in hepatocytes and liver mitochondria by their interference with mitochondrial electron transport chain. ${ }^{4-6}$ Contrary to oxidative stress, the role of nitrosative stress in cholestatic liver diseases has received much less attention. High levels of circulating bile salts during cholestasis disrupt intestinal mucosal barrier resulting in translocation of enteric bacteria to the mesenteric lymph nodes and the liver, ${ }^{7}$ and resulting endotoxemia is responsible for augmented nitric oxide (NO) synthesis by inducible NO synthase (iNOS). ${ }^{8,9}$ This excessive generation of NO has been observed both in experimental cholestasis ${ }^{8,10}$ and in primary biliary cirrhosis patients. ${ }^{9,10}$ The increase in hepatic and plasmatic circulating levels of NO and cytokines is determinant for the hepatocellular injury and the rapid progression of hepatic dysfunction in cholestatic settings.

There is a large body of evidence indicating that S-nitrosation of cysteine thiols (also known as S-nitrosylation) constitutes a significant route through which $\mathrm{NO}$ bioactivity is transduced. S-nitrosothiols (SNO), derived from proteins, peptides and amino acids, supply cellular compartments and extracellular fluids with NO bioactivity. Therefore, S-nitrosation has emerged as the prototype redoxbased post-translational modification ${ }^{11-13}$ and it has been proposed that disruption or deregulation of SNO signaling leads to impairment of cellular function and disease..$^{14}$ In this regard, we have previously shown the importance of the alteration of SNO homeostasis during hepatotoxin-induced cell death in human hepatocytes ${ }^{15}$ and demonstrated a definite control of SNO cellular content when high levels of S-nitrosoproteins are present in human hepatocytes. ${ }^{16}$

To date, there are scarce studies exploring the participation of S-nitrosation of proteins and SNO metabolism in hepatocellular cholestatic liver injury. Changes in circulating and hepatic levels of SNO have been recently reported in both bile duct-ligated rats ${ }^{10,17}$ and primary biliary cirrhosis patients. ${ }^{10}$ Consequently, interventions directed to favorably support NO metabolism in the early phase of cholestasis have been suggested to allow protein redox status maintenance. ${ }^{10}$ This study aimed to assess the role of $\mathrm{NO}$ synthesis, protein S-nitrosation and the alteration of SNO homeostasis in the triggering and development of hepatocellular injury during cholestasis induced by bile duct ligation (BDL) in rats. S-methylisothiourea (SMT) is a potent selective competitive iNOS inhibitor with a demonstrated efficacy in models in which this NOS isoform is involved, such as septic or hemorrhagic shock. ${ }^{18,19}$ Taurine-conjugated ursodeoxycholic acid (TUDCA) is known to protect hepatocytes in cholestasis by stimulating the expression and membrane insertion of transporter proteins. ${ }^{20,21}$ Therefore, we treated BDL rats with SMT, and TUDCA was used as the clinical comparator in these studies. Our results suggest that the inhibition of NO synthesis during induced cholestasis ameliorates hepatocellular injury, and that this therapeutic effect is in part mediated by the improvement of liver proficiency in maintaining SNO homeostasis.

\section{MATERIALS AND METHODS \\ Materials}

S-methylisothiourea hemisulfate was obtained from SigmaAldrich (St Louis, MO, USA) and TUDCA was from Calbiochem (San Diego, CA, USA). All other reagents were from Sigma-Aldrich unless stated otherwise.

\section{Animals and Surgical Procedures}

Male Wistar rats (200-250 g) were kept in controlled conditions of light (12-h light: 12-h dark cycle) and temperature $\left(22^{\circ} \mathrm{C}\right)$, and provided with food (Purina, Barcelona, Spain) and water ad libitum. The study was approved by the Research Committee of the Reina Sofia University Hospital. All animals received human care in compliance with the Spanish regulations and law. Rats were anesthetized with 3\% sevofluorane and the surgical procedure was initiated by a midline ventral incision with exposure of the common bile duct. Double ligature with silk suture were done and the bile duct was sectioned between them. Sham-operated (SO) rats were submitted to laparotomy and abdominal closure without bile duct intervention.

\section{Experimental Design}

Animals were randomly assigned to one of the four groups as follows: (i) sham-operated control group (SO group, $n=10$ ), (ii) bile duct-ligated group with a daily intraperitoneal injection of phosphate-buffered solution (PBS) (BDL group, $n=10$ ), (iii) BDL group with a daily intraperitoneal injection of $50 \mathrm{mg} / \mathrm{kg}$ TUDCA dissolved in PBS (TUDCA group, $n=10)$ and (4) BDL group with a daily intraperitoneal injection of $25 \mathrm{mg} / \mathrm{kg}$ SMT dissolved in PBS (SMT group, $n=10)$. In all, 10 animals in each group were killed 7 days after surgery under sevofluorane anesthesia. Blood was collected from the abdominal aorta, and the plasma and serum obtained were frozen at $-20^{\circ} \mathrm{C}$ until the measurement of biochemical parameters. After perfusion with cold saline solution, the liver was rapidly removed for biochemical and histological examination. Some liver specimens were frozen at $-80^{\circ} \mathrm{C}$ for the determination of S-nitrosoprotein content, S-nitrosoglutathione reductase (GSNOR) enzymatic activity, and Nos2 and Adh5 mRNA levels. Liver lysates were obtained by mechanical disruption of liver tissue (sample grinding kit, Roche, Madrid Spain) in lysis solution (50 mM Tris- $\mathrm{HCl}, \mathrm{pH}$ 7.4, $300 \mathrm{mM} \mathrm{NaCl}, 5 \mathrm{mM}$ EDTA, $0.1 \mathrm{mM}$ neocuproine, $1 \%$ Triton X-100 and $1 \mathrm{mM}$ PMSF plus aprotinin and leupeptin), incubating on ice for $15 \mathrm{~min}$ and centrifugation at $10000 \mathrm{~g}$ for $15 \mathrm{~min}$ at $4^{\circ} \mathrm{C}$. Supernatants were collected and protein was quantified with Bradford reagent (Bio-Rad, Hercules, CA, USA). Other liver specimens were fixed in formalin, processed in graded alcohol, xylene, and embedded in paraffin. Thin sections were obtained for evaluation of histological damage and immunohistochemical analyses. Hematoxylin-eosin and Masson's trichrome stainings were performed according to the standard procedure. 

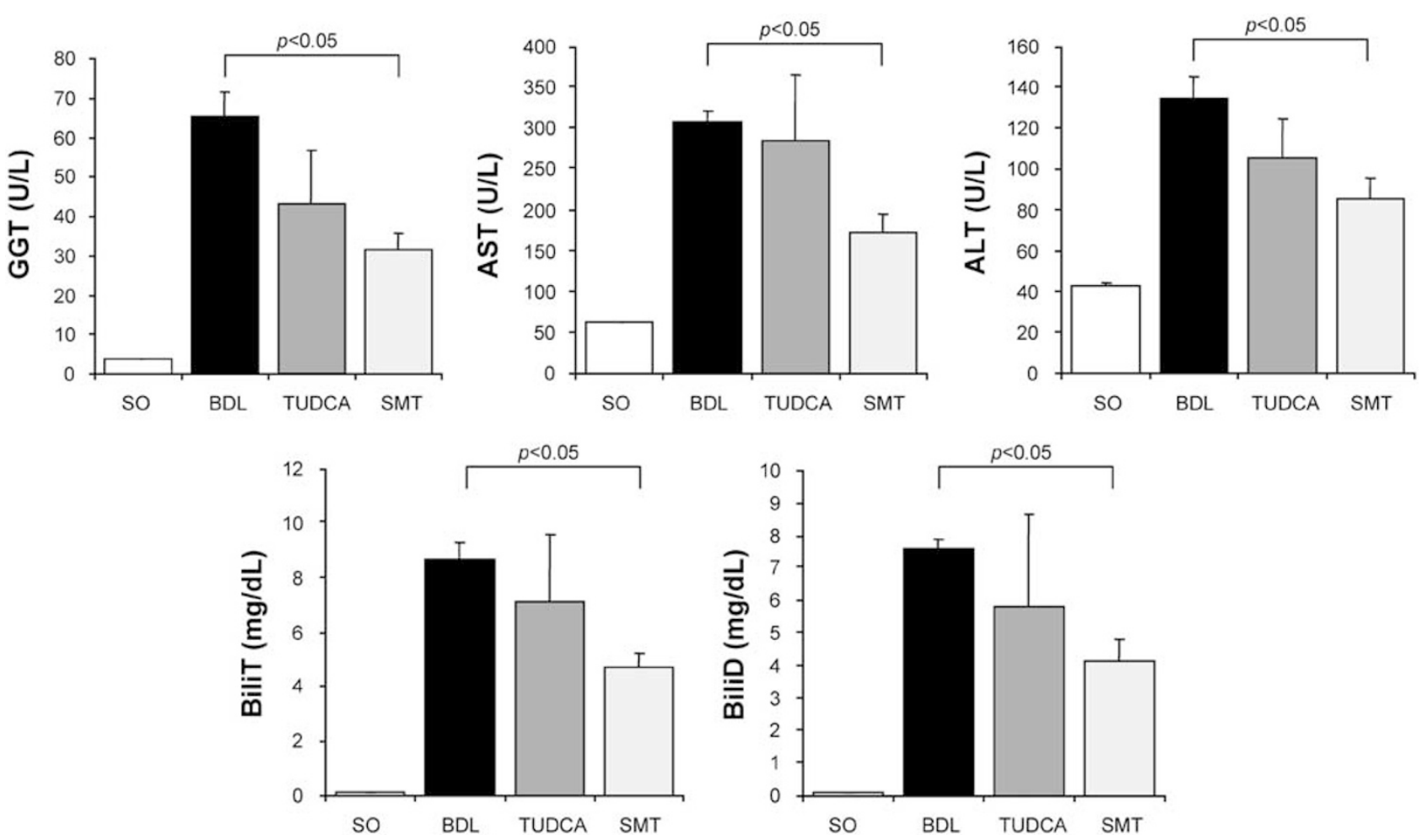

Figure 1 Serum markers of liver function in bile duct-ligated rats. Serum concentrations of aspartate aminotransferase (AST), alanine aminotransferase (ALT), $\gamma$-glutamiltranspeptidase (GGT), total (BiliT) and direct bilirubin (BiliD) were measured as indicators of hepatic injury in the different experimental groups. SO, sham-operated animals; BDL, animals subjected to bile duct ligation for 7 days without treatment; TUDCA (50 mg/kg) or SMT ( $25 \mathrm{mg} / \mathrm{kg}$ ), animals subjected to bile duct ligation for 7 days and administered daily with the indicated treatment. Data are means \pm s.e.m., $n=10$. For all the represented parameters, significant differences $(P<0.05)$ were observed comparing BDL, TUDCA or SMT with SO group, and no significant differences were obtained comparing BDL with TUDCA groups.

\section{Liver Biochemical Function Tests}

Serum concentrations of aspartate aminotransferase (AST), alanine aminotransferase (ALT), $\gamma$-glutamiltranspeptidase (GGT), total and direct bilirubin were measured as indicators of hepatic injury using standard diagnostic kits.

\section{Measurement of NO Production}

The NO stable end products nitrite plus nitrate $\left(\mathrm{NO}_{\mathrm{x}}\right)$ were measured in the plasma using a commercial kit (Total Nitric Oxide Assay Kit, Thermo Scientific, Rockford, IL, USA).

\section{Western Blot Analysis for Mrp2 Expression}

The expression of Mrp2 was determined by western blot analysis using a mouse monoclonal antibody (Santa Cruz Biotechnology). Immunoreactive bands obtained by autoradiography were quantified by densitometry (GS800 scanner, Bio-Rad) and analyzed by Quantity One software v.4.4.0 (Bio-Rad).

\section{GSNOR Activity}

S-nitrosoglutathione reductase activity was measured as described elsewhere. ${ }^{16}$ In brief, the liver lysates were incubated with $500 \mu \mathrm{M}$ GSNO and $200 \mu \mathrm{M}$ NADH in reaction buffer (20 mM Tris-HCl, pH 8.0, 0.5 mM EDTA) and absorbance at $340 \mathrm{~nm}$ was used to measure GSNO-dependent $\mathrm{NADH}$ consumption.

\section{Detection of Protein S-Nitrosation by the Biotin-Switch Method}

The procedure was performed as previously described. ${ }^{22}$ Briefly, the liver lysates were incubated with $20 \mathrm{mM}$ methyl methanethiosulfonate (Sigma) followed by acetone precipitation. Precipitates were centrifuged and resuspended in HENS buffer (250 mM HEPES pH 7.7, $1 \mathrm{mM}$ EDTA, $0.1 \mathrm{mM}$ neocuproine and $1 \%$ SDS) and then incubated with $1 \mathrm{mM}$ ascorbic acid and $4 \mathrm{mM} \mathrm{N}$-[6-(biotinamido)hexyl]$3^{\prime}$-(2'-pyridyldithio)propionamide (biotin-HPDP, Pierce, Rockford, IL, USA) for $1 \mathrm{~h}$. As biotin-HPDP is cleavable under the reduced conditions, prepared samples were loaded onto SDS-PAGE gels without dithiothreitol. All steps preceding SDS-PAGE were carried out in the dark. Biotinylated samples were then detected by western blot analysis using a primary monoclonal anti-biotin antibody (Sigma). 

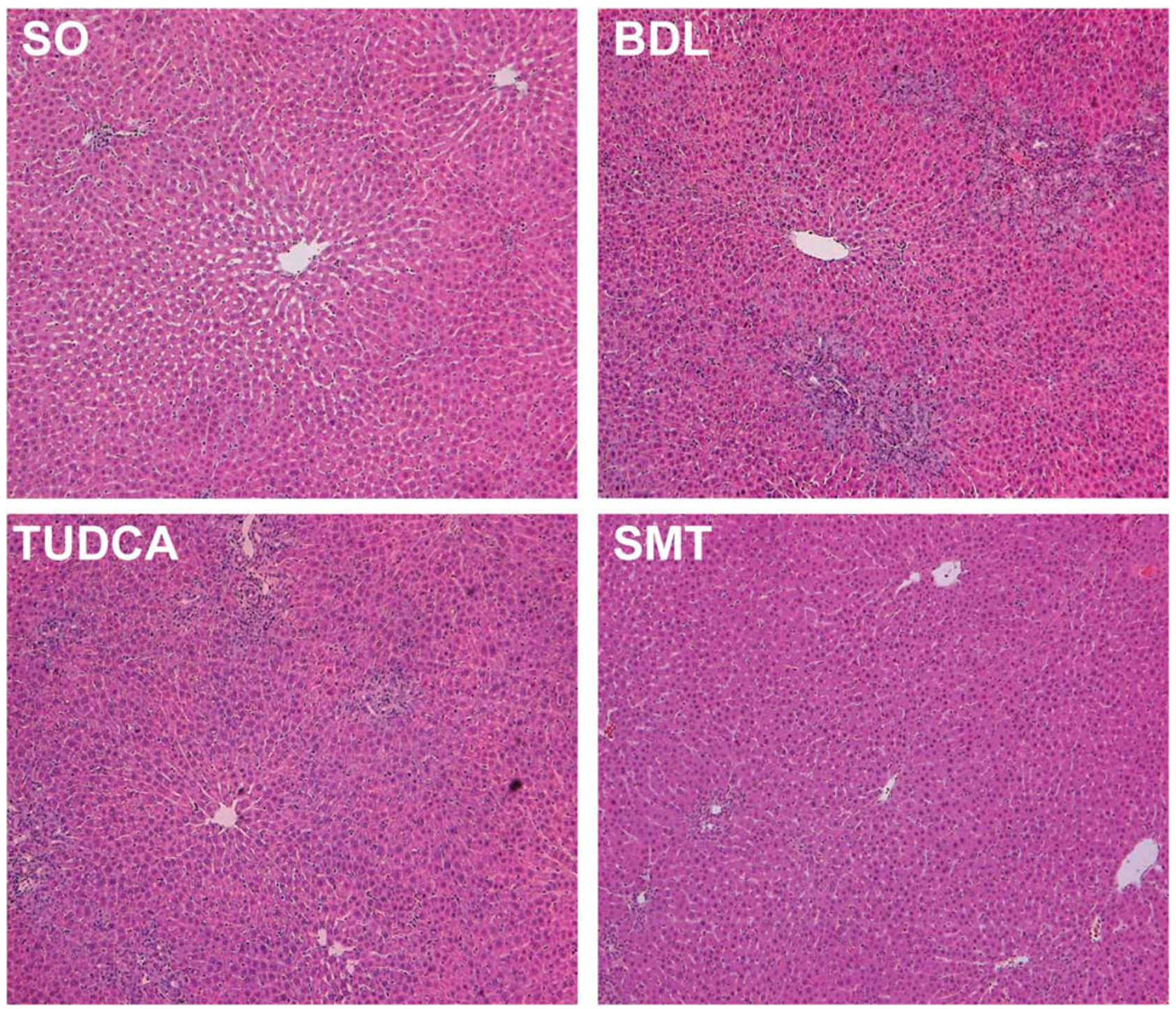

Figure 2 Histological analysis of liver damage. Evaluation of histological damage was carried out using hematoxylin-eosin stain in the liver tissue of animals from the different experimental groups. SO, sham-operated animals; BDL, animals subjected to bile duct ligation for 7 days without treatment; TUDCA $(50 \mathrm{mg} / \mathrm{kg})$ or SMT $(25 \mathrm{mg} / \mathrm{kg})$, animals subjected to bile duct ligation for 7 days and administered daily with the indicated treatment. Magnification: $\times 100$.

\section{Analysis of Nos2 and Adh5 mRNA by Quantitative Real-Time PCR}

Total RNA from hepatocytes was extracted using Trizol reagent according to the manufacturer's recommendations (Life Technologies). The expression of mRNA for Nos2 and Adh5 were examined by quantitative real-time RT-PCR using the LightCycler thermal cycler system (Roche Diagnostics, Indianapolis, IN, USA). RT-PCR was carried out in one step, using the QuantiTect SYBR Green RT-PCR kit (Qiagen GmbH, Hilden, Germany) following the manufacturer's protocol. The gene-specific primers used were: Nos2: 5' -AACCCAAGGTCTACGTTCAAG-3' (forward) and $5^{\prime}$-AAAGTGGTAGCCACATCCCG-3' (reverse); Adh5:
5'-CCACAGGCTCATGAAGTTCG-3' (forward) and 5'-GCC AACACTCTCCACAATGC- $3^{\prime}$ (reverse); and $\beta$-actin, $5^{\prime}$-TGA GAGGGAAATCGTGCGT- $3^{\prime}$ (forward) and $5^{\prime}$-TCATGGATG CCACAGGATTCC- $3^{\prime}$ (reverse). Results were normalized to that of $\beta$-actin. Quantification of relative expression was determined by the $2^{-\Delta(\Delta \mathrm{CT})}$ method. ${ }^{23}$

\section{Immunohistochemical Analysis}

Paraffin sections $(4 \mu \mathrm{m})$ on poly-L-lysine-coated slides were used after drying overnight at $37^{\circ} \mathrm{C}$. The sections were dewaxed in xylene, rehydrated in ethanol and incubated at $100^{\circ} \mathrm{C}$ in ChemMate Target Retrieval Solution pH 6.0 (Dako, Barcelona, Spain) for $20 \mathrm{~min}$. After washing in PBS, 
the sections were immunostained using an autostainer (Cytomation, Dako, Barcelona, Spain). Slides were incubated for $10 \mathrm{~min}$ in $3 \%$ hydrogen peroxide to block endogenous peroxidase, and then incubated $30 \mathrm{~min}$ with anticytokeratin 19 antibody (R\&D Systems, Madrid, Spain). After washing $5 \mathrm{~min}$ in PBS, the slides were incubated for $30 \mathrm{~min}$ with an HRP-labelled polymer (DAKO Envision System) and developed for $15 \mathrm{~min}$ using diaminobenzidine. Finally, the slides were counterstained with hematoxylin and mounted in Eukitt mounting medium.

\section{Quantitative and Semiquantitative Histological Analysis} Bile duct proliferation was scored in 12 randomly selected portal areas as absent, (0); mild (less than three bile ducts per portal space, 1 ); moderate (three to five bile ducts per portal space, 2); and severe (more than five bile ducts per portal space, 3). Cytokeratin 19-positive cells were counted in 12 randomly selected portal areas. The median was determined for portal space. Fibrosis was semiquantitatively scored on Massson's trichromic sections as absent (0), mild (1), moderate (2) or severe (3). Sections were independently scored by two observers. All histological studies were carried out by two observers unaware of the treatments.

\section{Purification of S-Nitrosoproteins}

Liver lysates (3-4 mg protein) were biotinylated as described above. Biotin-HPDP was removed by acetone precipitation and centrifugation, and the pellet was resuspended in HENS buffer as above. Two volumes of neutralization buffer (20 mM HEPES pH 7.7, $100 \mathrm{mM} \mathrm{NaCl}, 1 \mathrm{mM}$ EDTA and $0.5 \%$ Triton X-100) was added, and EZview ${ }^{\mathrm{TM}}$ Red streptavidin-agarose (Sigma) was added to purify biotinylated proteins. Biotinylated proteins were incubated with the resin for $1 \mathrm{~h}$ at room temperature, washed five times with neutralization buffer adjusted to $600 \mathrm{mM} \mathrm{NaCl}$, and then incubated with elution buffer $(20 \mathrm{mM}$ HEPES pH 7.7, $100 \mathrm{mM} \mathrm{NaCl}, 1 \mathrm{mM}$ EDTA and $100 \mathrm{mM}$ 2-mercaptoethanol) for $20 \mathrm{~min}$ at $37^{\circ} \mathrm{C}$ with gentle agitation to recover the bound protein. Supernatants were collected, and after separation in SDS-PAGE the protein bands were excised and trypsin digested.

\section{Protein Identification}

For the ESI-MS/MS analysis, microcapillary reversed-phase LC was performed with a CapLC ${ }^{\mathrm{TM}}$ (Waters, Milford, MA, USA) capillary system. Reversed-phase separation of tryptic digests were performed with an Atlantis, C18, $3 \mu \mathrm{m}$, $75 \mu \mathrm{m} \times 10 \mathrm{~cm}$ Nano Ease $^{\mathrm{TM}}$ fused silica capillary column (Waters) equilibrated in 5\% acetonitrile and $0.2 \%$ formic acid. After injection of sample $(18 \mu \mathrm{l})$, the column was washed during $5 \mathrm{~min}$ with the same buffer and the peptides were eluted using a linear gradient of $5-50 \%$ acetonitrile in $30 \mathrm{~min}$ at a constant flow rate of $0.2 \mu \mathrm{l} / \mathrm{min}$. The column was coupled online to a Q-TOF Micro (Waters) using a PicoTip nanospray ionization source (Waters). The heated capillary temperature was $80^{\circ} \mathrm{C}$ and the spray voltage was $1.8-2.2 \mathrm{kV}$. MS/MS data were collected in an automated data-dependent mode. The three most intense ions in each survey scan were sequentially fragmented by collision-induced dissociation using an isolation width of 2.5 and a relative collision energy of $35 \%$. Data processing was performed with MassLynx 4 and ProteinLynx Global Server 2 (Waters).

\section{Statistical Analysis}

Results are expressed as means with their corresponding standard errors. Comparisons were made using ANOVA with least significant difference test. Statistical significance was set at $P \leq 0.05$.

\section{RESULTS}

S-methylisothiourea treatment resulted in substantial, statistically significant reductions in serum levels of GGT, AST, ALT, and total and direct bilirubin in the obstructed animals (Figure 1). This striking profile demonstrates that SMT afforded marked hepatoprotection in this established model of extrahepatic cholestasis. It can be noted that SMT was much more effective in decreasing these markers of liver damage than TUDCA, which reduced them to some extent, but without statistical significance.

Histological analysis of liver sections using hematoxylineosin showed a loss of normal hepatic architecture with marked bile duct proliferation in portal areas in BDL animals. As shown in Figure 2, and according to the semiquantitative histological parameters described in the Materials and methods section, ductular proliferation was significantly reduced in SMT- (mild), but not TUDCA- (severe), treated animals. Immunohistochemical analysis to detect cytokeratin 19, a specific marker of mature cholangiocytes, confirmed that SMT treatment significantly reduced bile duct proliferation in obstructed rats. As shown in Figure 3, a significant lower number of CK19-positive cells was observed when compared with the BDL group $(57 \pm 10.8$ vs $10.6 \pm 1.4)$. The presence of fibrosis was evaluated in the Masson's trichromestained sections (Figure 4). The reduction of hepatic fibrosis was significantly more efficient after SMT (mild) than after TUDCA treatment (moderate), compared with the BDL group (severe).

The function of the canalicular conjugate export pump Mrp2 is impaired in various experimental models of cholestasis. To determine whether the expression of Mrp2 was altered at the protein level in our experimental model and by the TUDCA or SMT treatments, immunoblotting was performed on liver lysates. As shown in Figure 5, Mrp2 protein level was significantly downregulated in animals after 7 days of biliary obstruction compared with the SO group. Significantly, both TUDCA and SMT treatments returned the expression of this canalicular export pump to basal levels (Figure 5). 

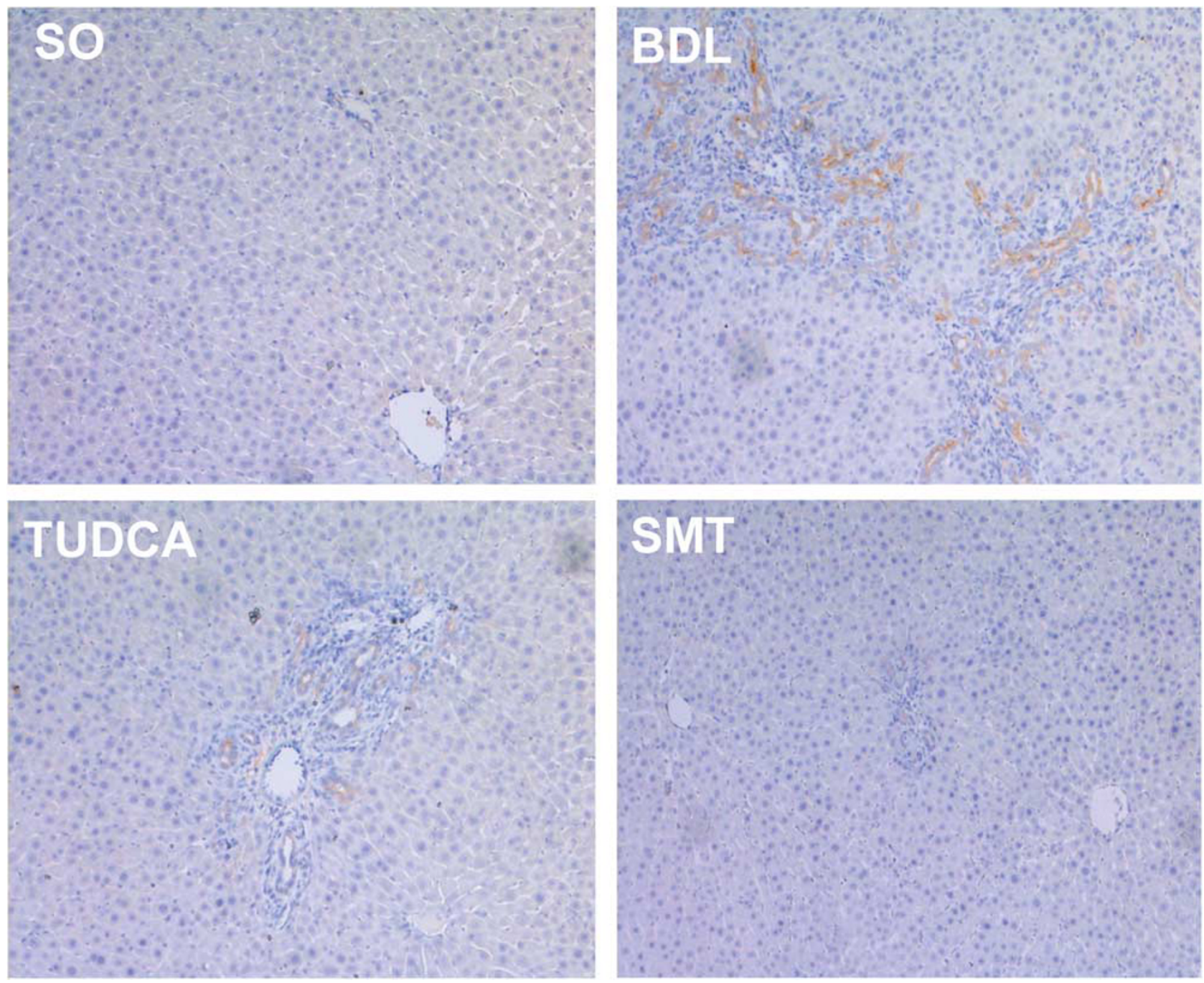

Figure 3 Immunohistochemical analyses of cytokeratin 19 expression. Immunodetection of cytokeratin 19 in the liver tissue of animals from the different experimental groups. SO, sham-operated animals; BDL, animals subjected to bile duct ligation for 7 days without treatment; TUDCA $(50 \mathrm{mg} / \mathrm{kg})$ or SMT $(25 \mathrm{mg} / \mathrm{kg})$, animals subjected to bile duct ligation for 7 days and administered daily with the indicated treatment. Magnification: $\times 100$.

Next, we investigated NO production by examining the levels of its stable end products, $\mathrm{NO}_{\mathrm{x}}$ in the plasma and hepatic Nos 2 mRNA expression levels. Circulating $\mathrm{NO}_{\mathrm{x}}$ levels were significantly higher the in BDL and TUDCA groups compared with control SO group. However, SMT treatment reduced NO production 7 days after BDL, as indicated by significantly reduced circulating $\mathrm{NO}_{\mathrm{x}}$ levels in the SMT group compared with the BDL group (Figure 6a). Quantitative RT-PCR analysis of Nos2 mRNA levels was carried out to explore the role of iNOS induction in augmented NO production in BDL rats. As shown in Figure 6b, obstructed animals showed significantly higher hepatic Nos2 mRNA levels than SO animals, but neither TUDCA nor SMT treatments significantly altered Nos2 induction after BDL.
To better define the mechanisms of the hepatoprotective effect of SMT in this model, we explored whether enhanced NO production in BDL rats altered the levels of hepatic S-nitrosoproteins. To this end, liver proteins were subjected to the biotin-switch assay that specifically biotinylates S-nitrosated proteins, which can then be detected by western blot. BDL increased the level of S-nitrosated proteins in the liver, and SMT was more effective in decreasing the enhanced S-nitrosation of hepatic proteins than TUDCA (Figure 7).

S-nitrosoglutathione reductase is an important metabolizing enzyme involved in SNO homeostasis. Therefore, we next explored whether BDL in rats may alter hepatic GSNOR activity. As shown in Figure 8a, GSNOR activity was 

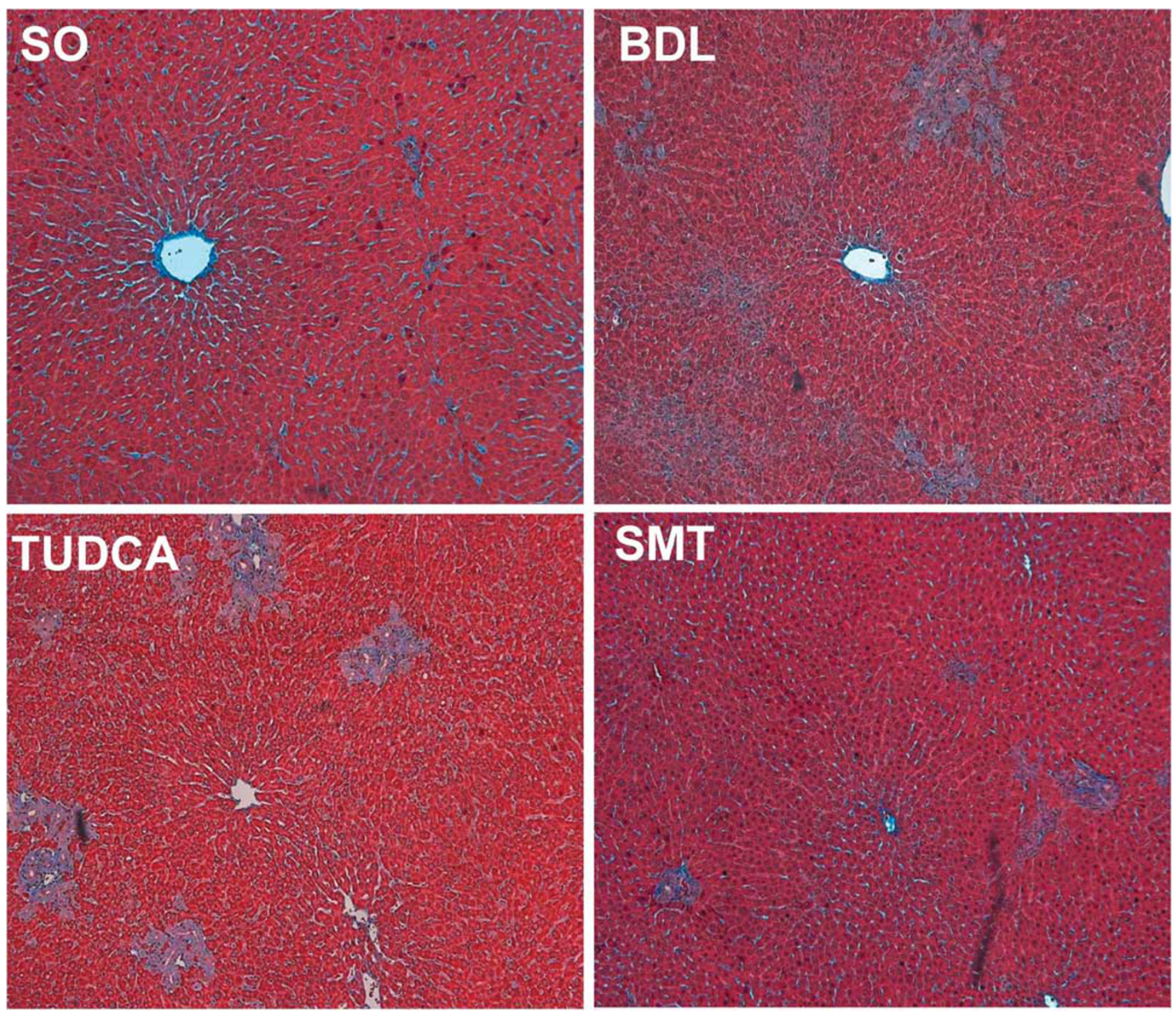

Figure 4 Histological analyses of liver fibrosis. Assessment of liver fibrosis was performed using Masson's trichrome stain in the liver sections from animals in the different experimental groups. SO, sham-operated animals; BDL, animals subjected to bile duct ligation for 7 days without treatment; TUDCA $(50 \mathrm{mg} / \mathrm{kg})$ or SMT $(25 \mathrm{mg} / \mathrm{kg})$, animals subjected to bile duct ligation for 7 days and administered daily with the indicated treatment.

Magnification: $\times 100$.

significantly downregulated in BDL rats. However, SMT, but not TUDCA, treatment returned the hepatic levels of this enzymatic activity to basal levels. Moreover, BDL induced a severe impairment in the liver expression of $A d h 5$, the gene encoding for rat GSNOR. TUDCA treatment was totally ineffective in the recuperation of $A d h 5$ expression levels. On the contrary, a remarkable recovery in Adh5 mRNA levels was observed in SMT-treated rats (Figure $8 \mathrm{~b}$ ).

To further explore the alteration of SNO homeostasis during cholestasis, the liver proteins that were S-nitrosated after bile duct ligation were purified and identified. To this end, following the biotin-switch method formerly
S-nitrosated liver proteins were purified using streptavidin-agarose, separated using SDS-PAGE and identified using ESI-MS/MS. In all, 25 proteins, including cytoskeleton proteins, metabolic and detoxification enzymes, and molecular chaperones were identified as S-nitrosoproteins in the liver of BDL rats, as revealed by this proteomics approach (Table 1). Among the identified proteins were the enzymes S-adenosyl methionine (AdoMet) synthetase, betainehomocysteine S-methyltransferase (BHMT), ATP-synthase, 3-ketoacyl-CoA thiolase, hydroxymethylglutaryl-CoA-synthase, and the molecular chaperones Hsp90 and protein disulfide isomerase. 


\section{DISCUSSION}

Cholangiocytes are considered biologically important epithelia because of the diverse array of cellular processes in

a

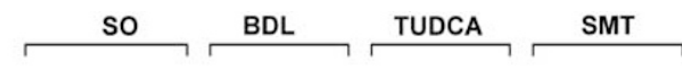

Mrp2

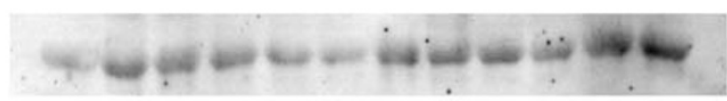

$\beta$-actin
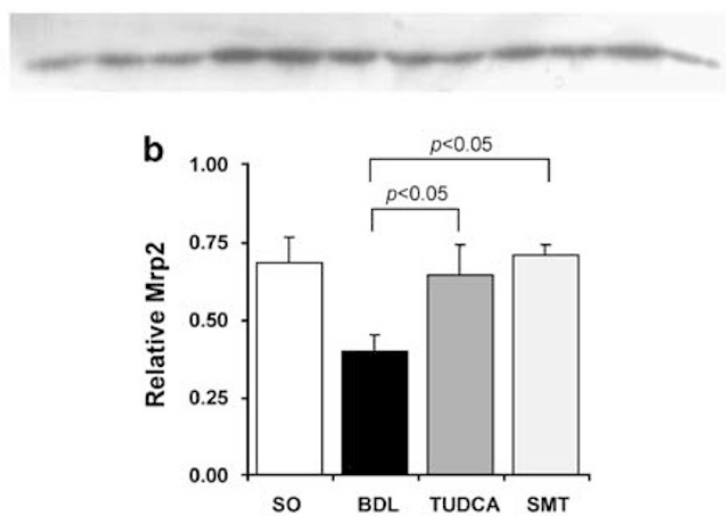

Figure 5 Hepatic Mrp2 protein expression in rats subjected to bile duct ligation. (a) Western blot analyses were carried out to analyze the Mrp2 pump protein expression in the livers of animals from the different experimental groups. The expression of $\beta$-actin was used as a control. SO, sham-operated animals; BDL, animals subjected to bile duct ligation for 7 days without treatment; TUDCA ( $50 \mathrm{mg} / \mathrm{kg}$ ) or SMT $(25 \mathrm{mg} / \mathrm{kg})$, animals subjected to bile duct ligation for 7 days and administered daily with the indicated treatment. The image is representative of three different experiments. (b) Corresponding densitometric analysis showing the ratio of Mrp2 to $\beta$-actin expression. Data are means \pm s.e.m. of three independent experiments. Significant diminished Mrp2 protein expression $(P<0.05)$ was observed comparing BDL with SO group, and no significant differences were obtained comparing TUDCA or SMT with SO group. which they participate, including transport of water, ions and solutes. ${ }^{24}$ The cholangiocytes have been proposed to be the principal target cell for bile acids in the liver, significantly altering cholangiocyte secretion, proliferation and survival. In this regard, it has been shown that bile acids can induce phosphorylation of EGF receptor promoting cell growth. ${ }^{25}$ Liver fibrosis during acute and chronic cholestasis involves the stepwise process of ductular reaction, which refers to an increasing number of ductules (that is, cholangiocyte proliferation) and an increase in matrix, leading to periportal fibrosis and eventually biliary cirrhosis. ${ }^{26}$ There is evidence that proliferating cholangiocytes have a role in the induction of fibrosis, either directly through epithelial-mesenchymal transition, ${ }^{27}$ or indirectly through the activation of hepatic stellate cells. ${ }^{28}$ In fact, targeting proliferating biliary epithelia might provide a novel antifibrotic therapy in the liver. ${ }^{29}$ Several studies have shown that the induction of iNOS and NO synthesis are also implicated in the promotion of cell growth in cholangiocytes. ${ }^{30,31}$ NO production is also one of the factors involved in the activation of hepatic stellate cells, the primary cell type responsible for the production of collagen I, the key protein involved in the development of liver fibrosis. ${ }^{32}$ Furthermore, the nitrosative stress caused by the NO has been implicated in the fibrotic changes of ethanol-induced hepatic liver fibrosis. ${ }^{33}$ Our study shows that the inhibition of NO synthesis caused a significant reduction of cholangiocyte proliferation after BDL in rats. Furthermore, the reduced grade of ductular reaction was accompanied by a reduced periportal fibrosis. Therefore, our results suggest that the chronic inhibition of NO synthesis may be considered as a useful therapy that may target cholangiocyte proliferation, reducing biliary fibrosis.
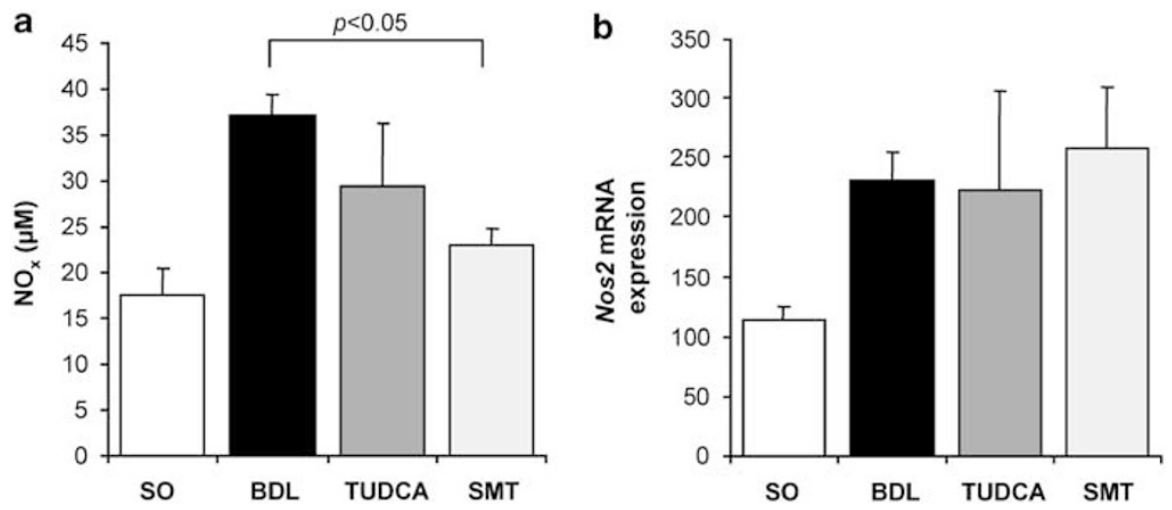

Figure 6 Nitric oxide (NO) production and hepatic Nos2 mRNA expression in bile duct-ligated rats. (a) The NO production was estimated by measuring in plasma its stable end products, nitrite plus nitrate $\left(\mathrm{NO}_{\mathrm{x}}\right)$. Data are means \pm s.e.m., $n=10$. Significant differences in $\mathrm{NO}_{\mathrm{x}}$ levels $(P<0.05)$ were observed comparing BDL or TUDCA with SO group, and no significant differences were obtained comparing TUDCA with BDL group or SMT with SO group. (b) The relative expression of liver mRNA for Nos2 was examined by quantitative real-time RT-PCR. SO, sham-operated animals; BDL, animals subjected to bile duct ligation for 7 days without treatment; TUDCA $(50 \mathrm{mg} / \mathrm{kg})$ or SMT $(25 \mathrm{mg} / \mathrm{kg})$, animals subjected to bile duct ligation for 7 days and administered daily with the indicated treatment. Data are means \pm s.e.m., $n=10$. Significant augmented Nos2 mRNA levels $(P<0.05)$ were observed comparing BDL, TUDCA or SMT with SO group. 

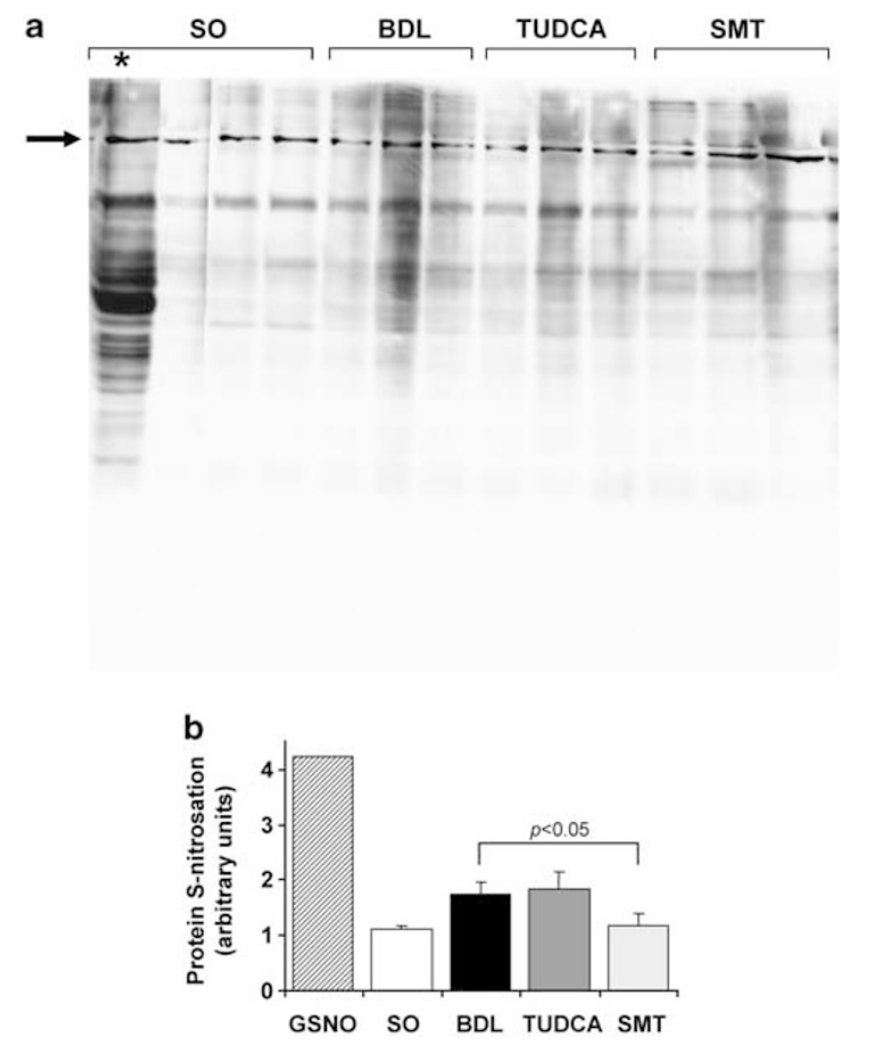

Figure 7 Detection of S-nitrosated proteins in the livers of bile ductligated rats. (a) Liver lysates of animals from the different experimental groups were subjected to the biotin-switch assay to detect S-nitrosoproteins. Asterisk indicates a positive control obtained by preincubating a liver lysate (SO group) with $200 \mu \mathrm{M}$ S-nitrosoglutathione (GSNO). SO, sham-operated animals; BDL, animals subjected to bile duct ligation for 7 days without treatment; TUDCA $(50 \mathrm{mg} / \mathrm{kg}$ ) or SMT ( $25 \mathrm{mg} / \mathrm{kg}$ ), animals subjected to bile duct ligation for 7 days and administered daily with the indicated treatment. The image is representative of three different experiments that included three animals of each experimental group. (b) Corresponding densitometric analysis of the total protein bands detected in the biotin-switch assay. The densitometry of the band corresponding to an endogenously biotinylated protein (arrow) detected in the assay was used as a loading control. The GSNO positive control is also included for comparison. Data are means \pm s.e.m. of three independent experiments. Significant augmented protein $S$-nitrosation $(P<0.05)$ was observed comparing $\mathrm{BDL}$ and TUDCA with SO group, and no significant differences were obtained comparing SMT with SO group.

The downregulation of Mrp2 expression has been observed in models of intrahepatic and obstructive cholestasis. ${ }^{34}$ TUDCA is known to stimulate the expression of transporter proteins and their targeting and insertion into the canalicular membrane for biliary secretion in the liver. ${ }^{20,21}$ In our study, treatment with TUDCA reverted Mrp2 protein expression to basal levels in obstructed animals. Remarkably, the inhibition of NO synthesis by treating the rats with SMT also caused a recovery in liver Mrp2 protein levels. It has been described that LPS-induced expression of iNOS is accompanied by a significant decrease of Mrp2 mRNA levels in rat liver slices. ${ }^{35}$ Accordingly, endotoxin-induced cholestasis in rat is mainly caused by an impaired canalicular secretion, with strong downregulation of Mrp2 and subsequent reduced bile salt secretion. ${ }^{36}$ Our results suggest that the therapeutic efficacy of SMT in obstructed animals can partially be explained by its effect on Mrp2 expression. The comparison of TUDCA treatment, which did not exert marked effect on NO production, with SMT treatment, which also increased Mrp2 expression, permitted us to explore additional molecular mechanisms in the hepatoprotection exerted by SMT.

S-substituted isothioureas inhibit the enzymatic activity of iNOS rather than its induction ${ }^{37}$ and others authors have shown that SMT inhibits NO production in the liver but not hepatic iNOS induction. ${ }^{38}$ Accordingly, our results show that in BDL rats the treatment with SMT, but not with TUDCA, significantly inhibited NO production and none of the two treatments significantly affected the induction of iNOS observed in ligated animals. These data support the hypothesis that the diminished synthesis of NO may explain the reduced nitrosative stress and the improved SNO metabolism observed in SMT-treated rats. It has been recently shown that chronic oral administration of UDCA decreases portal pressure, NO production and iNOS expression in BDL cirrhotic rats. ${ }^{39}$ In our model, the administration of TUDCA reduced NO production to some extent, although no effect was observed on Nos2 mRNA levels. Conceivably, the full effect of TUDCA on NO production and iNOS expression may only be observed when cirrhosis develops. Some of the protective effects of TUDCA that were observed in this study may also be related to the capability of TUDCA to moderately decrease NO production. Therefore, a combination of UDCA, or TUDCA, and SMT may have a notable effect on SNO homeostasis and hepatic function in BDL rats. However, this hypothesis needs to be explored in future studies.

Some studies have shown changes in circulating and hepatic levels of SNO in cholestatic conditions. ${ }^{10,17}$ We have previously shown that treatment of human hepatocytes with L-nitrosocysteine (CSNO) caused a rapid increase in S-nitrosoprotein content, ${ }^{15}$ returning later to basal levels due to an increase in GSNOR activity. ${ }^{16}$ This increased GSNOR activity in CSNO-treated hepatocytes seemed to be related to higher mRNA levels of Adh5, the gene encoding for human GSNOR, in these cells. ${ }^{16}$ Several studies have underlined the important role of GSNOR in the cellular defence against nitrosative stress. ${ }^{40,41}$ In this study, we have shown that BDL increased the hepatic S-nitrosoprotein content. Moreover, this augmented S-nitrosation of liver proteins in BDL rats was related to diminished GSNOR activity due to an impairment in the transcription of the rat gene encoding for this enzyme. The treatment of obstructed animals with SMT 

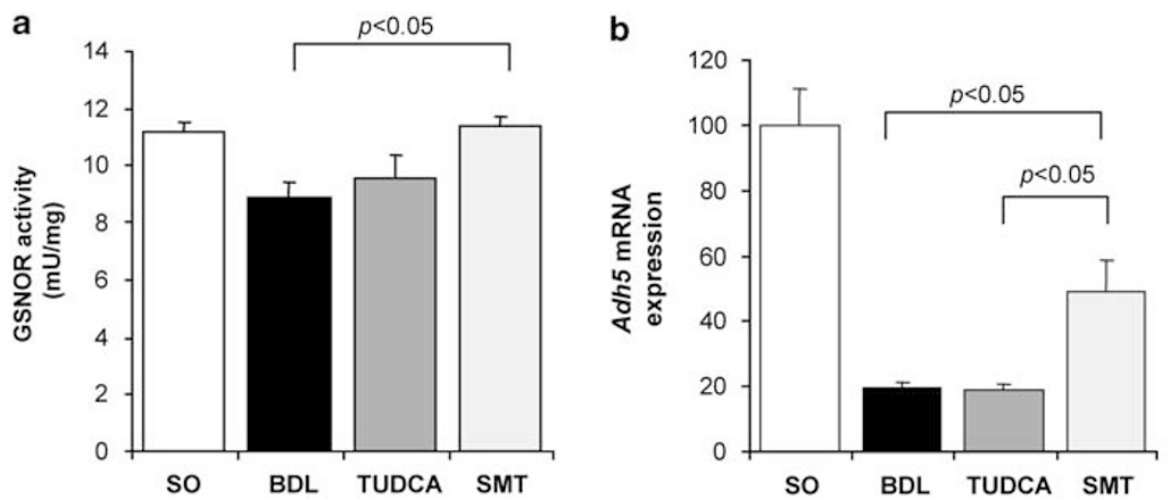

Figure 8 Hepatic S-nitrosoglutathione reductase (GSNOR) activity and Adh5 mRNA expression in bile duct-ligated rats. (a) GSNOR activity in the liver lysates was measured as the enzymatic GSNO-dependent NADH consumption. Data are means \pm s.e.m., $n=10$. Significant diminished GSNOR activity $(P<0.05)$ was observed comparing BDL or TUDCA with SO group, and no significant differences were obtained comparing TUDCA with BDL group or SMT with SO group. (b) The relative expression of the liver mRNA for Adh5, the gene encoding for GSNOR, was examined by quantitative real-time RT-PCR. SO, sham-operated animals; BDL, animals subjected to bile duct ligation for 7 days without treatment; TUDCA (50 mg/kg) or SMT (25 mg/kg), animals subjected to bile duct ligation for 7 days and administered daily with the indicated treatment. Data are means \pm s.e.m., $n=10$. Significant reduced Adh5 expression $(P<0.05)$ was observed comparing BDL, TUDCA, or SMT with SO group, and no significant differences were obtained comparing TUDCA with BDL group.

normalized hepatic GSNOR activity levels and reduced hepatic protein S-nitrosation. This normalization of SNO homeostasis was related to a remarkable recovery of Adh5 mRNA levels in obstructed rats treated with SMT. An adequate level of GSNOR activity is required to effectively metabolize GSNO, maintaining a proper equilibrium between GSNO and S-nitrosoproteins. Therefore, a diminished hepatic GSNOR activity in the context of elevated NO production may displace this equilibrium towards enhanced hepatic protein S-nitrosation, as observed in BDL- and TUDCA-treated rats. On the contrary, the beneficial effects of the iNOS inhibitor may be explained by the capability of SMT to reduce NO production and also to increase Adh5 expression and GSNOR activity, thus improving SNO homeostasis. SMT must be considered a relatively selective inhibitor of iNOS activity as, at least in vitro, it has shown its efficacy inhibiting the activity of purified human both iNOS and eNOS. ${ }^{42}$ Although the increased NO production in obstructed animals is mainly the result of iNOS induction, future studies with iNOS $(-/-)$ mice will undoubtedly help to clarify the precise role of both NOS isoforms in the alteration of $\mathrm{SNO}$ homeostasis during cholestasis.

Our proteomic analysis of S-nitrosated proteins in the liver of obstructed animals revealed important enzymes responsible for energy production, metabolism, as well as molecular chaperones and proteins involved in the structural integrity of the cells. The S-nitrosation of critical mitochondrial enzymes participating in ATP synthesis and fatty acid metabolism may explain the mitochondrial dysfunction and the disturbed fatty acid metabolism associated with cholestasis. ${ }^{43,44}$ It can be noted that two of the other identified proteins are important enzymes involved in the methionine cycle, namely, BHMT and AdoMet synthetase, also known as methionine adenosyltransferase (MAT). The inhibition of hepatic BHMT causes hyperhomocysteinemia, ${ }^{45}$ a condition that has been recently shown to be involved in hepatic fibrosis ${ }^{46}$ and is observed in primary billiary cirrhosis ${ }^{47}$ and obstructive cholestasis. ${ }^{48}$ The impairment of MAT function has been related to several liver conditions, such as cirrhosis, nonalcoholic steatohepatitis and hepatocellular carcinoma, and may contribute to liver disease pathogenesis. ${ }^{49}$ States of increased NO production, such as septic shock or hypoxia, induce MAT inactivation without affecting MAT gene expression. ${ }^{50}$ In fact, the S-nitrosation of hepatic MAT has been shown in vitro and in vivo, with the modification of Cys121 residue resulting in enzyme inactivation. ${ }^{50}$ The therapeutic benefit of AdoMet has been shown in the treatment of patients with intrahepatic cholestasis. ${ }^{49}$ Our results agree with the above-mentioned studies and suggest that BHMT and MAT may constitute hitherto unrecognized targets of nitrosative stress in cholestatic liver injury.

In summary, we have shown that the inhibition of NO production during induced cholestasis ameliorates hepatocellular injury, and that this effect is in part mediated by the improvement of cell proficiency in maintaining SNO homeostasis. Therefore, our results support the therapeutic benefit of inhibiting NO synthesis in a cholestatic setting and provide S-nitrosation targets that should facilitate future research in the development of new therapies in cholestatic liver diseases. 
Table 1 S-nitrosated proteins identified by LC-MS/MS analysis in BDL rat liver lysates

\begin{tabular}{|c|c|c|c|c|}
\hline Protein names & NCBI accession number & Number of sequenced peptides & Coverage (\%) & Loca \\
\hline \multicolumn{5}{|l|}{ Cytoskeleton proteins } \\
\hline$\alpha$-Actin-2 & P62738 & 1 & 10 & Cyt \\
\hline Tubulin $\beta$-2A chain & P04691 & 1 & 4 & Cyt \\
\hline \multicolumn{5}{|l|}{ Metabolic enzymes } \\
\hline S-adenosyl methionine synthetase type 1 & P13444 & 1 & 6 & Cyt \\
\hline Aldehyde dehydrogenase, microsomal & P30839 & 2 & 5 & ER \\
\hline ATP synthase, subunit- $\alpha$ & P15999 & 1 & 2 & Mit \\
\hline Betaine-homocysteine S-methyl-transferase 2 & Q68FT5 & 1 & 4 & Cyt \\
\hline Carbamoyl-phosphate synthase 1 & P07756 & 13 & 20 & Mit \\
\hline Carboxylesterase 3 precursor & P16303 & 3 & 7 & ER \\
\hline Dihydroxyacetone kinase & Q4KLZ6 & 2 & 6 & ER \\
\hline 10-formyltetrahydrofolate dehydrogenase & P28037 & 2 & 3 & Cyt \\
\hline Fructose-biphosphate aldolase B & P00884 & 3 & 11 & Cyt \\
\hline Glutamate dehydrogenase 1 & P10860 & 1 & 5 & Mit \\
\hline Hydroxymethylglutaryl-CoA synthase & P22791 & 3 & 17 & Mit \\
\hline 3-ketoacyl-CoA thiolase & P13437 & 1 & 8 & Mit \\
\hline L-lactate dehydrogenase A chain & P04642 & 1 & 6 & Cyt \\
\hline Long-chain fatty acid CoA ligase 1 & P18163 & 2 & 9 & Mit \\
\hline Malate dehydrogenase & P04636 & 1 & 21 & Mit \\
\hline Pyruvate carboxylase mitochondrial precursor & P52873 & 2 & 6 & Mit \\
\hline
\end{tabular}

Molecular chaperones

HSP 90- $\beta$

P34058

PDI

P04785

Ribophorin 1

Detoxification enzymes

Glutathione S-transferase Mu $1 \quad$ P04905

Glutathione S-transferase Mu 2

Signaling proteins

Elongation factor $1-\alpha 2$

Others

Serum albumin precursor
$\mathrm{P} 08010$

P62632

P02770
$\mathrm{P} 07153$

yt

Cyt

Cyt

R

Mit

Cyt

Mit

$R$

ER

Cyt

yt

Mit

Mit

Mit

Cyt

Mit

Mit Mit

1

2

2

6

Cyt

9 ER

$7 \quad \mathrm{ER}$

\section{cation}


4. Reinehr R, Graf D, Haussinger D. Bile salt-induced hepatocyte apoptosis involves epidermal growth factor receptor-dependent CD95 tyrosine phosphorylation. Gastroenterology 2003;125:839-853.

5. Sokol RJ, Winklhoferroob BM, Devereaux MW, et al. Generation of hydroperoxides in isolated rat hepatocytes and hepatic mitochondria exposed to hydrophobic bile-acids. Gastroenterology 1995;109:1249-1256.

6. Sokol RJ, Mckim JM, Goff MC, et al. Vitamin E reduces oxidant injury to mitochondria and the hepatotoxicity of taurochenodeoxycholic acid in the rat. Gastroenterology 1998;114:164-174.

7. Assimakopoulos SF, Scopa CD, Charonis A, et al. Experimental obstructive jaundice disrupts intestinal mucosal barrier by altering occludin expression: beneficial effect of bombesin and neurotensin. J Am Coll Surg 2004;198:748-757.

8. Arriero MM, Lopez-Farre A, Fryeiro $\mathrm{O}$, et al. Expression of inducible nitric oxide synthase in the liver of bile duct-ligated Wistar rats with modulation by lymphomononuclear cells. Surgery 2001;129:255-266.

9. Hokari $A$, Zeniya $M$, Esumi $H$, et al. Detection of serum nitrite and nitrate in primary biliary cirrhosis: possible role of nitric oxide in bile duct injury. J Gastroenterol Hepatol 2002;17:308-315.

10. Grattagliano I, Portincasa P, Palmieri VO, et al. Mutual changes of thioredoxin and nitrosothiols during biliary cirrhosis: results from humans and cholestatic rats. Hepatology 2007;45:331-339.

11. Stamler JS, Lamas S, Fang FC. Nitrosylation. the prototypic redoxbased signaling mechanism. Cell 2001;106:675-683.

12. Mannick JB, Schonhoff CM. Nitrosylation: the next phosphorylation? Arch Biochem Biophys 2002;408:1-6.

13. Martinez-Ruiz A, Lamas S. S-nitrosylation: a potential new paradigm in signal transduction. Cardiovasc Res 2004;62:43-52.

14. Foster MW, McMahon TJ, Stamler JS. S-nitrosylation in health and disease. Trends Mol Med 2003;9:160-168.

15. Lopez-Sanchez LM, Collado JA, Corrales FJ, et al. S-Nitrosation of proteins during $\mathrm{D}$-galactosamine-induced cell death in human hepatocytes. Free Radic Res 2007;41:50-61.

16. Lopez-Sanchez LM, Corrales FJ, Gonzalez R, et al. Alteration of $\mathrm{S}$-nitrosothiol homeostasis and targets for protein S-nitrosation in human hepatocytes. Proteomics 2008:8:4709-4720.

17. Ebrahimkhani MR, Kiani S, Oakley F, et al. Naltrexone, an opioid receptor antagonist, attenuates liver fibrosis in bile duct ligated rats. Gut 2006;55:1606-1616.

18. Szabo C, Southan GJ, Thiemermann C. Beneficial effects and improved survival in rodent models of septic shock with S-methylisothiourea sulfate, a potent and selective inhibitor of inducible nitric oxide synthase. Proc Natl Acad Sci USA 1994;91:12472-12476.

19. Bazzani C, Bertolini A, Guarini S. Inhibition of nitric oxide synthases enhances the effect of ACTH in hemorrhagic shock. Life Sci 1997;61:1889-1897.

20. Fickert $P$, Zollner $G$, Fuchsbichler $A$, et al. Effects of ursodeoxycholic and cholic acid feeding on hepatocellular transporter expression in mouse liver. Gastroenterology 2001;121:170-183.

21. Beuers U, Bilzer M, Chittattu A, et al. Tauroursodeoxycholic acid inserts the apical conjugate export pump, Mrp2, into canalicular membranes and stimulates organic anion secretion by protein kinase C-dependent mechanisms in cholestatic rat liver. Hepatology 2001;33:1206-1216.

22. Lopez-Sanchez LM, Corrales FJ, De la MM, et al. Detection and proteomic identification of S-nitrosated proteins in human hepatocytes. Methods Enzymol 2008;440:273-281.

23. Livak KJ, Schmittgen TD. Analysis of relative gene expression data using real-time quantitative PCR and the 2(-Delta Delta $C(T)$ ) method. Methods 2001;25:402-408.

24. Bogert PT, LaRusso NF. Cholangiocyte biology. Curr Opin Gastroenterol 2007;23:299-305.

25. Werneburg NW, Yoon $\mathrm{JH}$, Higuchi $\mathrm{H}$, et al. Bile acids activate EGF receptor via a TGF-alpha-dependent mechanism in human cholangiocyte cell lines. Am J Physiol Gastrointest Liver Physiol 2003;285:G31-G36.

26. Roskams T, Desmet V. Ductular reaction and its diagnostic significance. Semin Diagn Pathol 1998;15:259-269.

27. Diaz R, Kim JW, Hui JJ, et al. Evidence for the epithelial to mesenchymal transition in biliary atresia fibrosis. Hum Pathol 2008;39:102-115.
28. Omenetti A, Porrello A, Jung $Y$, et al. Hedgehog signaling regulates epithelial-mesenchymal transition during biliary fibrosis in rodents and humans. J Clin Invest 2008;118:3331-3342.

29. Patsenker E, Popov Y, Stickel F, et al. Inhibition of integrin alphavbeta6 on cholangiocytes blocks transforming growth factor-beta activation and retards biliary fibrosis progression. Gastroenterology 2008;135:660-670

30. Ishimura N, Bronk SF, Gores GJ. Inducible nitric oxide synthase upregulates cyclooxygenase-2 in mouse cholangiocytes promoting cell growth. Am J Physiol Gastrointest Liver Physiol 2004;287: G88-G95.

31. Ishimura N, Bronk SF, Gores GJ. Inducible nitric oxide synthase up-regulates Notch-1 in mouse cholangiocytes: implications for carcinogenesis. Gastroenterology 2005;128:1354-1368.

32. Vera $M$, Nieto N. Hepatic stellate cells and alcoholic liver disease. Rev Esp Enferm Dig 2006:98:674-684.

33. Nanji AA, Jokelainen K, Lau GK, et al. Arginine reverses ethanolinduced inflammatory and fibrotic changes in liver despite continued ethanol administration. J Pharmacol Exp Ther 2001;299:832-839.

34. Trauner $\mathrm{M}$, Arrese $\mathrm{M}$, Soroka $\mathrm{CJ}$, et al. The rat canalicular conjugate export pump (Mrp2) is down-regulated in intrahepatic and obstructive cholestasis. Gastroenterology 1997;113:255-264.

35. Elferink MG, Olinga P, Draaisma AL, et al. LPS-induced downregulation of MRP2 and BSEP in human liver is due to a posttranscriptional process. Am J Physiol Gastrointest Liver Physiol 2004;287: G1008-G1016.

36. Vos TA, Hooiveld GJ, Koning $H$, et al. Up-regulation of the multidrug resistance genes, Mrp1 and Mdr1b, and down-regulation of the organic anion transporter, Mrp2, and the bile salt transporter, Spgp, in endotoxemic rat liver. Hepatology 1998;28:1637-1644.

37. Southan GJ, Szabo C, Thiemermann C. Isothioureas: potent inhibitors of nitric oxide synthases with variable isoform selectivity. $\mathrm{Br} \mathrm{J}$ Pharmacol 1995;114:510-516.

38. Olinga $\mathrm{P}$, Merema MT, de Jager $\mathrm{MH}$, et al. Rat liver slices as a tool to study LPS-induced inflammatory response in the liver. J Hepatol 2001;35:187-194.

39. Yang YY, Huang YT, Lee KC, et al. Chronic administration of ursodeoxycholic acid decreases portal pressure in rats with biliary cirrhosis. Clin Sci (Lond) 2009;116:71-79.

40. Liu L, Hausladen A, Zeng M, et al. A metabolic enzyme for S-nitrosothiol conserved from bacteria to humans. Nature 2001;410: 490-494.

41. Liu L, Yan Y, Zeng M, et al. Essential roles of S-nitrosothiols in vascular homeostasis and endotoxic shock. Cell 2004;116:617-628.

42. Garvey EP, Oplinger JA, Tanoury GJ, et al. Potent and selective inhibition of human nitric oxide synthases. Inhibition by non-amino acid isothioureas. J Biol Chem 1994;269:26669-26676.

43. Krahenbuhl L, Schafer M, Krahenbuhl S. Reversibility of hepatic mitochondrial damage in rats with long-term cholestasis. J Hepatol 1998;28:1000-1007.

44. Lang C, Berardi S, Schafer M, et al. Impaired ketogenesis is a major mechanism for disturbed hepatic fatty acid metabolism in rats with long-term cholestasis and after relief of biliary obstruction. J Hepatol 2002;37:564-571.

45. Collinsova M, Strakova J, Jiracek J, et al. Inhibition of betainehomocysteine S-methyltransferase causes hyperhomocysteinemia in mice. J Nutr 2006;136:1493-1497.

46. Zou CG, Gao SY, Zhao YS, et al. Homocysteine enhances cell proliferation in hepatic myofibroblastic stellate cells. J Mol Med 2009;87:75-84.

47. Biagini MR, Tozzi A, Marcucci R, et al. Hyperhomocysteinemia and hypercoagulability in primary biliary cirrhosis. World J Gastroenterol 2006;12:1607-1612.

48. Ebrahimkhani MR, Sadeghipour H, Dehghani M, et al. Homocysteine alterations in experimental cholestasis and its subsequent cirrhosis. Life Sci 2005;76:2497-2512.

49. Mato JM, Lu SC. Role of S-adenosyl-L-methionine in liver health and injury. Hepatology 2007;45:1306-1312.

50. Avila MA, Corrales FJ, Ruiz F, et al. Specific interaction of methionine adenosyltransferase with free radicals. Biofactors 1998;8:27-32. 